\title{
Langues en contact, langues en danger : une étude de cas autour de la négation verbale et autres mots- $\mathrm{N}$ en francoprovençal des Pouilles
}

\author{
Daniela, Puolato \\ Université de Naples Federico II \\ daniela.puolato@unina.it
}

Résumé. Les minorités francoprovençales de Faeto et Celle di San Vito (Pouilles, Italie) présentent l'intérêt d'un espace sociolinguistique qui permet d'étudier la variation linguistique dans une situation de langues en contact englobant une langue en danger. Menacé d'extinction, le francoprovençal est en contact, dans ces villages, avec l'italien et plusieurs dialectes centre-méridionaux relevant du groupe linguistique italo-roman. L'article propose une analyse micro-variationniste de la négation verbale en faetano-cellese. Ces deux variétés de francoprovençal ne connaissent qu'un seul marqueur de négation, l'adverbe pa (pas). Cependant, la présence dans la phrase d'autres éléments à valeur négative (adverbiaux ou nominaux) entraîne une certaine variabilité du fait que l'expression du marqueur négatif est optionnelle. L'analyse s'appuie sur trois différentes typologies d'énoncés francoprovençaux : écrits, élicités par traduction de l'italien et d'oral semi-spontané. La configuration syntactico-sémantique analysée touche une zone de variabilité potentielle du système gallo-roman et en même temps un point de résistance du système faetano-cellese aux pressions assimilatrices des autres langues de contact, ce qui amène à réfléchir sur la nature et les causes de la variabilité qui se produit dans une langue non standardisée et en voie d'extinction.

\begin{abstract}
The Francoprovençal minority languages of Faeto and Celle di San Vito (Puglia, Italy) represent an interesting socio-linguistic space for the study of language variation in a context of languages in contact which includes an endangered language. Threatened with extinction, Francoprovençal is in contact-in both countries-with Italian and several central and southern dialects belonging to the Italo-Romance linguistic group. This article deals with a micro-variational analysis of verbal negative forms in the languages spoken in Faeto and Celle. These two varieties of Francoprovençal show only one negative marker, the adverb pa (pas). However, the presence of other negative elements in the sentence (either adverbial or nominal) causes some variability, as the occurrence of the negative particle is optional. The analysis is based on three different typologies of Francoprovençal utterances: the written ones, the ones elicited by translation from Italian, and the semi-spontaneous spoken ones. The analyzed syntactic-semantic configuration involves a range of potential variability of the Gallo-Romance system and, at the same time, it represents a resistance of the language system of Faeto and Celle to the assimilation pressure of other languages in contact. This leads us to reflect on the nature and the reasons of the variability that occurs in a nonstandardized and endangered language.
\end{abstract}




\section{Introduction}

Les situations de langues en contact posent la question, très délicate, de la difficulté de distinguer la variation intrasystémique (due aux trajectoires évolutives d'une langue) de la variation intersystémique (due au contact entre les langues) ${ }^{1}$. En effet, d'une part, les mécanismes de changement sont essentiellement les mêmes, et, d'autre part, le contact linguistique n'explique pas tous les changements caractérisant une langue, étant donné qu'ils peuvent résulter de dynamiques internes de la langue. Cela vaut également pour la variation observable dans les langues en danger ou obsolescentes, à l'égard desquelles la différence semble tenir avant tout au grand nombre de changements qui les affectent et à la rapidité avec laquelle ils se produisent. S'il est prévisible que le processus d'obsolescence d'une langue passe par l'appropriation massive de patterns et de formes propres aux autres langues en contact avec elle, le degré d'influence que celles-ci exercent sur la langue en danger dépend à la fois du niveau de compétence de ses locuteurs et des modalités de coexistence des langues entre elles. En effet, l'un des aspects les plus cruciaux dans l'étude des changements linguistiques dus au contact est l'interrelation de facteurs sociaux et structurels ${ }^{2}$. Selon la durée et l'intensité du contact, les langues en danger tendent à adopter de nombreuses formes lexicales de la langue dominante, mais aussi des éléments morphosyntaxiques, des marques discursives et stylistiques: «Before passing into extinction, an obsolescent language may become a "carbon copy" of the dominant idiom » (Aikhenvald, $2012: 102$ ).

Ce processus de convergence de la langue en danger vers la langue dominante peut brouiller son affiliation génétique (Aikhenvald, 2012). Le rôle des traits typologiques caractérisant les langues en contact a fait aussi l'objet d'une étude menée dans le cadre d'une approche multi-causale du changement (Epps, 2012). La plus ou moins grande distance génétique ou typologique entre les langues peut influencer le degré de perméabilité réciproque des systèmes linguistiques, mais la proximité spatiale entre les variétés est susceptible, elle aussi, d'entraîner des changements typologiques, d'où l'importance d'études reliant typologie et dialectologie (Bisang, 2004 : 14 ; Miestamo, 2011 : 83-84).

L'analyse des phénomènes d'obsolescence linguistique se complique davantage du fait que, pour bon nombre de langues, il n'existe pas de descriptions suffisamment détaillées et complètes, en synchronie comme en diachronie. C'est justement le cas des deux variétés francoprovençales, à savoir le faetano et le cellese, parlées respectivement dans les minorités linguistiques de Faeto et Celle di San Vito (Pouilles, Italie). Le francoprovençal (FP) y est parlé depuis 800 ans environ. Cependant, suite aux bouleversements socio-économiques qui se sont produits tout au long des siècles et de la pression exercée par la langue nationale, le FP est aujourd'hui une langue « en danger » ${ }^{3}$. Les minorités francoprovençales de Faeto et Celle présentent l'intérêt d'un espace sociolinguistique qui permet d'étudier la variation linguistique dans un contexte de contact de langues englobant une langue en danger. Menacé d'extinction, le FP des Pouilles, appartenant au groupe gallo-roman, est en contact avec l'italien (IT) et plusieurs dialectes centre-méridionaux (campaniens, apuliens, abruzzais, molisans) relevant du groupe linguistique italoroman. Le contact prolongé avec l'IT et les dialectes italo-romans parlés sur le territoire a eu des répercussions principalement sur le lexique et la phonétique du FP. En revanche, il semble que la morphosyntaxe maintienne encore ses caractères propres, dont par exemple la négation verbale en $p a$ (pas) (Nagy, 2011). 
Notre étude consiste en une analyse micro-variationniste de la négation verbale en faetano-cellese, tout particulièrement des phrases négatives impliquant d'autres éléments à valeur négative, adverbiaux ou nominaux. Après une introduction centrée sur le phénomène linguistique et le système linguistique concernés par l'étude - la négation et le FP, l'accent sera mis sur la situation d'enquête et sur la description des structures négatives en faetano et cellese, issues d'un corpus de terrain dont nous définirons les caractéristiques. Les résultats de cette analyse ferons, ensuite, l'objet d'une synthèse comparative portant sur l'interrelation entre les structures négatives repérées et les différentes typologies de données qui composent le corpus.

\section{La négation : remarques liminaires}

Dans le World Atlas of Language Structures (WALS) (Haspelmath et al., 2005), Dryer (2011a, b, c) présente une analyse détaillée de la phrase négative standard d'un point de vue typologique. Pour former une phrase négative, la plupart des langues du monde ont recours à une particule négative placée avant ou après le verbe (Dryer, 2011c). Des constructions avec deux ou trois particules négatives sont aussi possibles (Dryer, 2011b). Généralement, lorsque la construction négative comporte deux marqueurs de négation, ceux-ci entourent le verbe. La négation discontinue ne...pas du français standard (FS) est un exemple de ce genre de construction ${ }^{4}$. La négation préverbale est le type le plus fréquent parmi les langues inclues dans le WELS, suivie par le type où l'élément de négation est postposé au verbe (carte 143A du WELS). En ce qui concerne les cas de négation discontinue, les données du WELS montrent que la négation double obligatoire est plus fréquente que la construction discontinue où l'un des deux éléments négatifs est facultatif (carte 143A du WELS).

Dès qu'il est question de phrase négative, la référence à la théorie de Jespersen (1917) devient incontournable. Par l'expression «cycle de Jespersen» (Dahl, 1979), on désigne le processus diachronique qui est à l'origine d'une négation double. Selon l'idée exprimée par Jespersen (1917:4) et, avant lui, par Meillet (1912 : 393 [1926 : 140]), un deuxième mot négatif est ajouté pour renforcer l'«adverbe négatif original » dont la valeur négative s'est affaiblie avec le temps. Il peut arriver que ce mot négatif supplémentaire soit conçu comme l'élément négatif à part entière et qu'il soit affecté, au fil du temps, par la même évolution du mot négatif initial et le «cycle », ou cette " sorte de développement en spirale » (Meillet, 1912 : 394 [1926 : 139-140]), recommence. Le passage d'une négation verbale en ne préposée à la forme finie du verbe de l'ancien français (je ne sais) à une négation double en ne...pas (je ne sais pas) du FS moderne, et en pas postverbal du français moderne non standard (je sais pas), représente l'exemple le plus connu du cycle de Jespersen. Ce dernier prévoit donc trois phases évolutives, à savoir NégV > NégVNég > VNég, mais d'autres modèles évolutifs insistent sur l'existence de stades intermédiaires et sur le fait que les structures associées aux différentes étapes peuvent aussi coexister à un moment donné. Van der Auwera, Vossen et Devos (2013) distinguent la période d'un « français plus ancien », où la négation verbale se fait en ne et ne...pas, de celle du français moderne qui dispose des trois constructions en ne, ne...pas et pas. L'évolution de la négation en français donnerait donc lieu à un «scénario étendu » par rapport à celui de base, comportant non plus trois étapes mais quatre ou cinq ${ }^{5}$. Généralement, ce type de changement est analysé comme le résultat d'une dynamique interne au système linguistique n'ayant aucun rapport avec le contact interlinguistique. Ce dernier semble toutefois jouer un rôle significatif par rapport à la position des marqueurs négatifs et, par conséquent, au positionnement d'une langue dans le cycle de Jespersen (Heine et Kuteva, $2003: 530$ ).

Dans les langues dites à concordance négative $(\mathrm{CN})$, la co-occurrence d'un marqueur négatif et d'un mot$\mathrm{N}^{6}$ est interprétée sémantiquement comme une seule négation (personne ne vient; non viene nessuno). L'IT et le FS exploitent différemment le phénomène de la CN. L'IT et les dialectes de l'Italie du Sud (Corblin et Tovena, 2003) sont des langues à CN généralisée, étant donné que l'association du marqueur avec un mot-N produit toujours une lecture de concordance. Le FS possède, en revanche, la particularité de formuler la négation phrastique au moyen d'un morphème discontinu et celle d'être une langue à $\mathrm{CN}$ restreinte. Les mots-N, associés au co-négatif ne dans les constructions à verbe tensé, peuvent participer à une chaîne de $\mathrm{CN}$ sans donner lieu, dans un contexte non marqué, à une lecture de double négation, alors 
que le marqueur pas est exclu du système de la CN (Corblin et Tovena, 2003). Par rapport au FS, il existe des variétés, dont le français québécois, le créole martiniquais (Déprez, 2003) ou le picard (Dagnac, 2015), qui présentent différentes conditions de compatibilité entre le marqueur pas et le(s) mot(s)-N avec le(s)quel(s) il interagit dans le contexte de la phrase. Il devient alors intéressant d'analyser ce genre de configuration syntactico-sémantique dans une variété gallo-romane en danger, qui subit, à différents niveaux et à différents degrés, l'influence de langues non gallo-romanes.

\section{Le francoprovençal : une « langue dialectale »}

Si, du temps où il était le vernaculaire des populations de la France centre-orientale, de la Suisse romande et du Val d'Aoste, le FP n'a coïncidé avec aucune entité politique précise, il n'a même pas connu de processus de standardisation, de koinésation ou reçu une dénomination unifiante. L'autonomie du type linguistique lui-même a longtemps fait l'objet de disputes. D'ailleurs, l'appellation « franco-provençal » (Ascoli, 1878), avec trait d'union, «fait penser immédiatement à une langue mixte, faite en partie de français et en partie de provençal » (Tuaillon, 1983: 5). Le fait est que, tout en partageant des traits communs avec les langues d'oc et d'oül, le FP constitue un système linguistique avec ses propres particularités et une «entité génétique à part entière » ${ }^{7}$ (Chambon et Greub, $\left.2000: 174\right)$, dont Hasselrot (1938/1939) a précisé les limites géographiques ${ }^{8}$.

On relie l'origine et le sort du FP à ceux de la ville de Lyon. Carrefour des grandes voies romaines de l'Occident et capitale des Gaules, Lyon a été le centre d'irradiation des innovations qui sont à la base du FP, mais qui préludent aussi à la formation du français ${ }^{9}$. À partir du moment où, vers la fin du Moyen Âge, Lyon perd son rôle de centre hégémonique et adopte le français, l'histoire du FP se confond avec un processus de marginalisation et fragmentation géolinguistique. Le morcellement des parlers francoprovençaux en une myriade de patois, qui leur confère un caractère éminemment « dialectal» (Tuaillon, 1988 : 191), est le signe précurseur de la tendance de ces parlers à être des langues en danger.

En France et en Suisse, le nombre des locuteurs de FP est, en effet, en très forte régression. En revanche, dans le Val d'Aoste, le FP conserve une plus grande vitalité. Le FP survit encore dans les minorités de Faeto et Celle, fondées au $13^{\mathrm{e}}$ siècle par un groupe de soldats angevins provenant de la France sudorientale, plus précisément des départements de l'Isère et de l'Ain (Melillo, 1959), peut-être de deux villages différents (Martino, $2011: \mathrm{XV}$ ).

Par leur histoire même, les variétés francoprovençales s'inscrivent pleinement dans la problématique des langues en danger tant sur le plan diachronique que synchronique.

\subsection{La négation dans les parlers francoprovençaux : quelques données}

Afin de mettre davantage en valeur la singularité du système de la négation en faetano-cellese par rapport aux parlers francoprovençaux de la France, de la Suisse romande et de la Vallée d'Aoste, nous proposons quelques données sur le fonctionnement de la négation dans ces parlers. Cette démarche permet de mieux vérifier, dans la mesure du possible, les effets éventuels du contact entre ces variétés dialectales et leurs langues officielles, le FS et l'IT. Pour ce faire, nous nous appuyons sur un corpus d'environ 300 énoncés négatifs, équitablement repartis parmi les trois territoires, et tirés d'une sélection restreinte, faite au hasard, de textes de nature, d'âge et de sources diverses ${ }^{10}$. Bien conscients du manque de représentativité de ce corpus, nous le considérons comme une sorte d' "échantillon de contrôle » à l'égard du corpus apulien.

Deux tendances se dégagent de manière nette : les patois valdôtains n'emploient que le pas (dont les variantes grapho-phonétiques dans nos textes sont pa/pàlpo/pó), tandis que les parlers des régions transalpines présentent l'alternance des structures avec et sans la particule ne. Le type ne...pas (ne...pâ/pô/pòs/pôs/pâo/pe) est le plus fréquent (69\% et 56\%), mais dans les patois de la Suisse romande, le pourcentage des structures en pas/pa/pâ/pâo/po est plus élevé (44\% contre 31\%). Le même polymorphisme syntaxique se vérifie dans les tournures avec un adverbe négatif, dont jamais 
(jaméldjamâldjaméldzamais) et plus (plu/plié/pllie/pielpus/pâ méi) sont de loin les plus fréquents, ou avec un pronom négatif, personne (sous la variante nion) et rien (sous les varaintes rin, ryin, ran,). En patois valdôtain, les structures sont $\mathrm{V}+$ jaméljamì, $\mathrm{V}+$ pamé/pamì/pomé/pomì ; les formes des pronoms sont gneun et ren. Dans cinq cas seulement, il y a co-occurrence du marqueur pa et d'un mot-N, à savoir avec méi, guéro, rente, més ren et gneun ${ }^{11}$. Les marqueurs et les autres mots-N sont antéposés au verbe lorsque celui-ci est à la forme infinitive.

Le polymorphisme syntaxique qui constitue l'alternance ne...pas/pas n'est qu'un exemple de la variabilité intrinsèque aux systèmes linguistiques considérés, qui l'héritent de leur propre parcours évolutif de langues à tradition orale, non standardisées, sans koïné. Plus généralement, le polymorphisme qui caractérise les parlers francoprovençaux semble donner lieu à une « variation libre », sans corrélation avec des catégories sociolinguistiques, mais qui «dans le cadre de l'évolution linguistique » pourrait « être réaffectée et réutilisée de manière fonctionnelle » (Diémoz et Kristol, 2012:178 ). Cette variabilité touche également le FP de Faeto et Celle, mais on verra par la suite qu'elle se manifeste sous des aspects divers tant au niveau lexical que syntaxique.

\subsection{Le corpus faetano-cellese}

Le FP apulien a fait l'objet, ces dernières années, d'une politique linguistique de protection, dont la mise en place d'activités visant à une standardisation de la langue, comme par exemple la production de grammaires et dictionnaires. On ne peut s'empêcher de tenir compte de ce genre de production du fait qu'écrire en cellese-faetano signifie aussi doter la langue de textes écrits qui «serviront de modèle, [...] c'est l'équiper, la normaliser, l'illustrer, la construire » (Bavoux, 2003 : 31). De plus, dans un contexte de contact de langues, l'écrivain et le lexicographe, en fonction de leurs choix linguistiques en tant qu'usager et descripteur de la langue, peuvent contribuer à raidir ou assouplir les «frontières » entre les langues en contact (Bavoux, 2003 : 32). Grâce à la politique linguistique de ces dernières années, à Faeto et Celle une première «frontière » a été effacée, du moins dans les représentations linguistiques des locuteurs (Puolato, 2010, 2011, 2013, 2016), à savoir entre langue et dialecte : le FP est aujourd'hui considéré comme une «langue » à égalité avec l'IT. Mais, nombreux sont les modes et les modalités par lesquels des «frontières » de toute autre nature s'écroulent dans les productions de nos locuteurs (phénomènes de code-switching, de code-mixing, d'emprunts, etc.) et dont les locuteurs ne sont que partiellement conscients. C'est pourquoi, il est intéressant d'examiner des typologies de données variées.

Les données écrites de notre corpus sont tirées de l'anthologie Les Cunte d'ajére é d'avi (Genovese et Pastore, 2011), relative à l'édition 2010 du Premio "Giuseppe La Nave » ${ }^{12}$. Cette anthologie a fait l'objet de notre choix puisqu'elle introduit la traduction cellese en regard des textes écrits en faetano.

Le corpus oral se constitue de données recueillies auprès de 55 locuteurs (29 Faetani et 26 Cellesi) à partir de la traduction d'une liste de phrases de l'IT au FP et de productions semi-spontanées en FP (de brèves conversations). Les locuteurs ont été répartis en trois classes d'âge : la I ${ }^{\text {ère }}$ comprend les adolescents et jeunes adultes (de 12 à 35 ans), la II ${ }^{\text {ème }}$ inclut les adultes âgés (entre 36 et 60 ans) et la III $^{\text {ème }}$ les personnes ayant plus de 60 ans. Les trois tranches d'âge indiquées comportent, tendanciellement, différents profils de locuteurs. La I ${ }^{\text {ère }}$ et la II ${ }^{\text {ème }}$ classes impliquent des «semilocuteurs » (Dorian, 1977), mais aussi quelques «jeunes locuteurs traditionnels » (Dorian, 1981), la III ${ }^{\text {ème }}$ classe se constitue surtout de «locuteurs traditionnels» (Dorian, 1981). Dans les contextes de contact de langues, la compétence linguistique des locuteurs est un paramètre d'analyse jugé pertinent lorsqu'un changement linguistique est en cours (Aikhenvald, $2012: 77$ ).

L'accès au corpus tout entier s'appuie sur un critère d'analyse statistique, du fait que le degré de récurrence d'une forme linguistique est une dimension constitutive du corpus lui-même. Sur la base d'une estimation quantitative de la fréquence d'apparition des structures négatives impliquant ou non différents types de mots-N, il convient de faire une distinction entre structures négatives dominantes (dont les fréquences d'apparition atteignent plus de 60\%), structures négatives secondaires (ayant un taux 
d'occurrence inférieur à 60\%) et structures négatives exceptionnelles (produites seulement par quelques locuteurs). La description des structures envisagées fait l'objet du paragraphe qui suit.

\subsection{Description de la négation en faetano-cellese}

En IT, outre l'usage du marqueur non, une phrase peut être niée ultérieurement par la présence d'un ou plusieurs mots-N. Les phrases négatives à traduire en FP se caractérisent par la présence de mots-N adverbiaux, associés au marqueur non et placés en position postverbale, ou nominaux, associés ou non au marqueur négatif, selon leur position par rapport au verbe. Lorsqu'un ou plusieurs mots-N s'associent à non, celui-ci est préposé à la forme fléchie du verbe et le ou les mots-N doivent être placés en position postverbale (non vedo nessuno; non dirò nulla). Dans le registre oral, non est parfois omis même si le mot-N est postposé au verbe (siamo mica scemi ou fa niente). S'il n'y a qu'un seul mot-N et s'il précède le verbe, le marqueur négatif est exclu (nessuno dovrà saperlo). Lorsqu'il y a deux ou plusieurs mots-N, l'un d'entre eux précède le verbe, tandis que l'autre ou les autres le suivent ${ }^{13}$ (nessuno compra niente).

Dans l'illustration qui suit, les énoncés sont organisés par type de structure, mais la référence aux différents sous-corpus est maintenue en ajoutant aux exemples les sigles CE (Corpus Écrit, 225 énoncés négatifs), CT (Corpus de Traduction, environ 700 phrases négatives) ${ }^{14}$ et CO (Corpus Oral, 196 énoncés négatifs).

La négation totale constitue un type de négation dominante dans le corpus écrit autant que dans le corpus oral. Elle se forme avec pa postposé à la forme fléchie du verbe (ex. 1-2) ou entre l'auxiliaire et le participe passé dans les formes composées du verbe (ex. 3-4). Il n'existe aucun cas de négation bipartite, ni d'emploi du seul co-négatif ne. En général, $p a$ apparaît dans des contextes divers, déclaratifs avant tout, mais aussi interrogatifs (ex. 5), ou avec un verbe modal (ex. 6-7) ${ }^{15}$ :

1. A-gn-ave 'na vàje un marì é 'na meglije che i tenevànte pa fiàue. (G\&P : 24)- $\mathrm{CE}^{16}$

'Il était une fois un mari et sa femme qui n'avaient pas d'enfant'

2. gi ge gióche pa (FMI4) ${ }^{17}-\mathrm{CO}$

'moi, je (ne) joue pas'

3. Ó parescìve ch'éve pa passà un menùte che m'éve checjà che ge sentì ciantà lu alle. (G\&P : 54) - CE

'J'avais l'impression que ça faisait à peine une minute que j'étais couché que le coq s'est mis à chanter

4. gi me s'éve pa [abbitu'wa]/(abbetuà) (CFI2) - CO

'je (ne) m'étais pas habituée'

5. pecché $?^{18}$ te la $[\mathrm{de}] /($ dénne) pa? (FFI3) - $\mathrm{CO}$

'pourquoi ? il (ne) va pas te la (une demi-heure) donner ?'

6. i ulevànte pa gettà pe l'àrje tant'anne de sacreficje (G\&P : 32) - CE

'ils ne voulaient pas jeter toutes ces années de sacrifices en l'air'

7. pecché i puó pa satre (FFIII) - CO

'parce qu'il (ne) peut pas sortir'

$53 \%$ des négations totales du corpus oral sont construites avec les verbes étre (ou éte) (34\%), fa (faire) $(15 \%)$, savàjere (savoir) (15\%), piàtre (plaire) $(13 \%)$, dirre (dire) $(9 \%)$, allà (aller) $(8 \%)$, avàjere (avoir) $(6 \%)$. Plus de la moitié des énoncés négatifs émis par les locuteurs sont donc construits autour d'un nombre réduit de verbes très fréquents et présentent une syntaxe minimale sujet-verbe :

8. auì a-étte próprje na bèlla giurnà, demmànne ge sé pa cóme ó vate a cunghìje (CFIII4) - CO 'aujourd'hui, c'est une belle journée, je (ne) sais pas comment ce sera demain'

9. ne ${ }^{19}$ sun partì da Cèlle pecché tràje [kud'dzino]/(seràue) cunzepprìne) mìnne i savavànte pa né [sor'rento] tùtte la ['kosta amalfi'tanə] (CFII2) - CO

'on est partis de Celle parce que trois cousines à moi (ne) connaissaient ni Sorrente ni la Côte amalfitaine'

10. chjénze anne lu stésse cartíje me pjà pa (FMI1) - CO

'quinze ans au même endroit, ça (ne) me plaît pas' 


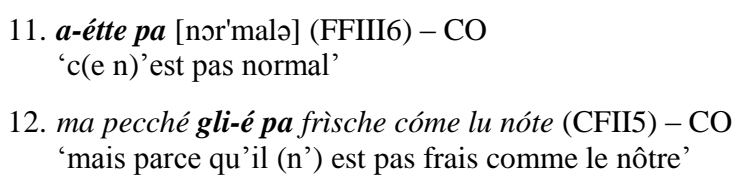

Tout comme en IT et dans les dialectes italo-romans, les verbes aux modes infinitif (ex. 13-14) et gérondif (ex. 15) sont précédés de nun :

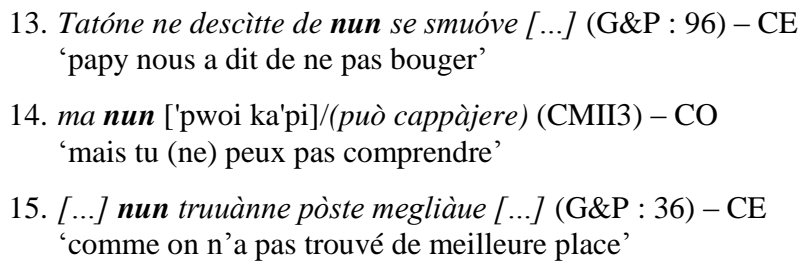

Une forte incertitude entoure la traduction de la phrase Quanto vorrei non aver corso così tanto ! (Qu'estce qui m'a pris de courir autant !) du corpus de traduction, probablement à cause de la complexité de la structure verbale formée d'un verbe modal conjugué au conditionnel et suivi d'un infinitif passé à la forme négative. En faetano-cellese, le conditionnel est souvent confondu avec le subjonctif (ex. 17 : avísse) si bien que ces deux modes peuvent s'employer l'un à la place de l'autre (SLF, 2007b : 287). De plus, le subjonctif est souvent remplacé par l'indicatif, au présent ou au passé composé (SLF, 2007b : 285). Nos énoncés attestent aussi l'emploi de l'imparfait (ex. 16 : ulíve). En effet, les locuteurs ont choisi, soit le subjonctif, soit l'imparfait ou ils ont opté pour des reformulations s'éloignant nettement de la structure verbale de la phrase source: par exemple chi me l'a fé fa i cùrrere accussì tàn CFII3. En général, la négation a été le plus souvent déplacée de l'infinitif au verbe modal :

16. canne ge ulíve pa cuórre accussì namùrre (FMI3) -

'Qu'est-ce qui m'a pris de courir autant !'

17. canne ge avísse pa uli cùrrere namùrre (CFII2) - CT

'Qu'est-ce qui m'a pris de courir autant!'

On voit apparaître la forme a pa dans les tournures négatives avec un verbe à la forme impérative :

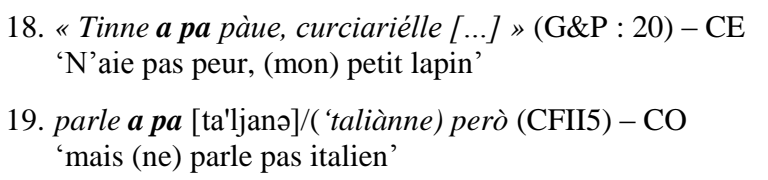

Martino (2011 : 50) précise que pa est remplacé par l'adverbe gin/ginne (gint dans Stich, 1998 : 94) (pas du tout) lorsque le verbe est esserci (y avoir) employé à la forme négative et avec la particule pronominale ne (en) (il donne l'exemple suivant: Aiat lu pan ? N'aiat gin / Vi è del pane ? Non ve n'è / Y a t-il du pain ? Il n’y en a pas). Toutefois, dans le Dizionario francoprovenzale di Faeto (SLF, 2007a), l'emploi de ginne (non ne en IT) ne semble lié qu'à la présence de la particule ne (ge uóglie gin / non ne voglio / je n'en veux pas), comme c'est le cas de l'énoncé tiré de notre corpus :

\section{0. addùnche sólde se fasciavànt 'ngìnnh (CMIII3) - CO}

'à l'époque, l'argent, on (n') en gagnait pas'

En ce qui concerne les structures négatives impliquant des mots-N adverbiaux, tels que nemmeno (même pas, pas même), mai (jamais) et più (plus), deux tendances sont à signaler : l'optionalité du marqueur et le fait que certains mots-N, comme par exemple chjù (plus), màje (jamais), manghe (pas même), témoignent par leur forme du contact avec l'IT et les dialectes italo-romans. La plupart des énoncés ayant un mot-N appartiennent au corpus de traduction et la tendance la plus importante consiste en l'emploi du mot-N tout seul. Ce type de structures négatives constitue une tendance secondaire dans les corpus écrit et oral. Le mot-N se place après le verbe fini ou après l'auxiliaire lorsque le verbe est conjugué à un temps composé. 
La traduction des structures du type non $+A u x+$ più $+V$ et les textes d'oral semi-spontané mettent en lumière le fait qu'à la forme chjù en faetano correspond l'emploi de pa ou de pa...méje en cellese. Cette différence ne ressort pas des textes écrits caractérisés par l'emploi généralisé de chjù. La négation s'appuyant sur le mot-N chjù représente une tendance secondaire dans le corpus écrit et son occurrence est sporadique dans le corpus oral :

21. La Funtàne du paìje gli-éste chjù [...] (G\&P : 124) - CE

'il n'y a plus la Fontaine du pays'

22. ge t'é chjù vijàue (FMI5) - CT

'je (ne) t'ai plus vu'

23. ge véje chjù (FFIII6) - CO

'je (ne) vais plus'

24. me sé pa resaglí méje a [mə mettə y kumunəka'tsjuyə] do sò quattrà ne sun sta [m va'kançə] (CMIII3) - CT

' $\mathrm{j}(\mathrm{e} \mathrm{n})$ 'ai pas réussi à me mettre en contact avec les garçons qu'on a connus en vacance'

25. ge t'é pa vijàue méje [n ḑirə] (CFIII2) - CT

'je (ne) t'ai plus vu'

Pour ce qui est de la traduction des tournures du type non $+V+$ mai ou non $+A u x+m a i+V$, l'utilisation de màje comme élément unique de négation fait office de négation dominante ; la locution manghe na vàje (pas une seule fois) peut être choisie au lieu de màje (ex. 28). Quelques exemples sont aussi présents dans le corpus d'oral semi-spontané :

26. g'é màje vijàue tante crestiànne a ciavù (FMI2) - CT

'j(e n)' ai jamais vu autant de monde chez vous'

27. ne lesciùnte màje da sule (FMI3) - CT

'on (ne) nous laisse jamais seuls'

28. ne lesciùnte manghe na vàje sule (FFIII3) - CT

'on (ne) nous laisse jamais seuls'

29. ló sise ge lò mìnge màje (FFII1) - CO

'les pois chiches, j'en mange jamais'

30. ge l'é màje pràje ci S. (CFII5) - CO

'je (ne) l'ai jamais pris chez S.'

La structure du type non $+V+$ nemmeno est réalisée, le plus souvent, avec l'adverbe manche/manghe, parfois substitué par nemméne. Manghe est la variante dialectale de manco, forme quasi-exclusive de l'IT parlé contemporain, utilisée à la place des adverbes neanche, nemmeno et neppure de l'IT standard :

31. Ge tenìve accussì pàue che ge resaglìve manghe a alluccà [...] (G\&P : 96) - CE

'J'avais tellement peur que je n'arrivais même pas à crier'

32. ne sun arrevà manche a la metà de la vi che gi già me sé stànche (CFI1) - CT

'nous (ne) sommes même pas à mi-chemin et je me sens déjà fatiguée'

L'emploi du seul marqueur négatif caractérise les corpus écrit et oral, tandis que l'emploi des seuls mots$\mathrm{N}$ chjù, màje et manghe caractérise le corpus de traduction. En revanche, la co-occurrence du marqueur et d'un mot-N constitue toujours une tendance secondaire, bien que plus représentée dans le corpus oral :

33. G'éve pa màje viàue tante crestiànne é tante puttéje denghjé Faìte. (G\&P : 52) - CE

'Je n'avais jamais vu autant de monde et autant de commerces à Faeto'

34. ma seràue s'é pa màje màje se [Jkarpo]/(scàrepe ) (CFI2) -

'ma sœur n'a jamais mis ces chaussures'

35. pecché la scóle icchì gli-éste pa méje (CMII2) - CO

'parce qu'(il n') y a plus d'école ici'

36. [...] se i tardive a arrevà $i$ vulive pa manghe mengìje. (G\&P : 104) $-\mathrm{CE}$ 
'quand il arrivait en retard, il ne voulait même pas manger

37. lu savùnte pa mànche a Fógge che ne sistùnnh (CFI2) - CO 'même à Foggia y savent pas qu'on existe'

Le corpus oral renferme aussi des énoncés où le marqueur s'associe à l'adverbe d'insistance próprjelprópje, variante dialectale de l'IT proprio (justement, exactement, vraiment) :

38. pure le vì i sunte pa própje bunne (FFII4) - CO

'les routes non plus sont pas très bonnes'

39. sélle ge la minge pa próprje (CFI2) - $\mathrm{CO}$

'celle-là, je la mange même pas'

Une tendance mineure consiste à effacer le mot-N et à n'employer que le marqueur négatif :

40. $m$ 'é pa resaglì a [mə mettə $\mathrm{y}$ kun'dattə] dò lò quattrà che sun sta a la [va'kanckə] (FMII2) - CT ' $\mathrm{j}(\mathrm{e} \mathrm{n})$ 'ai pas réussi à contacter les garçons qu'on a connus en vacance'

41. ge t'é pa vijàue [in dzira] (FMI4) - CT 'je (ne) t'ai pas vu'

42. g'é pa vijàue [tandə]/(tante) crestiànne a ciaù (CMIII3) - CT 'j(e n)'ai pas vu autant de monde chez lui'

43. se lasciùnte pa [im pafð]/('mpace) (CMI1) - CT 'ils (ne) nous laissent pas tranquilles'

44. ma seràue i-a pa màje se scàrepe (FFII4) - CT 'ma sœur n'a pas mis ces chaussures'

45. nusse ne sunne pa arrevà a metà de la ciarriére e gjà me s'é stanghe (CFII1) - CT 'nous (ne) sommes pas à mi-chemin et je me sens déjà fatiguée'

Les structures peu fréquentes, ou dont l'occurrence est tout à fait sporadique, sont aussi intéressantes. Elles montrent, par exemple, que la forme chjù, qu'on peut considérer comme spécifiquement faetana selon les données de traduction, peut être employée également par des locuteurs cellesi, mais elle se trouve associée à méje. La négation au moyen de chjù méje constitue une négation renforcée par l'emploi de deux mots-N supposés sémantiquement identiques (ex. 46-47). Il est aussi à noter que manghe peut être antéposé au verbe, probablement pour des raisons d'emphase (ex. 48-49).

46. ge t'é chjù vijàue méje [in ḑ̉irə] (CFII6) - CT 'je (ne) t'ai plus vu'

47. [pa'pa]/(tatà), gli-éste chiù méje addùnche ne fasciùnne chiù méje la zùppe (CFII4) - CO 'papa (n') est plus là, alors nous (ne) faisons plus de soupe'

48. mànche se fasciavànt 'struzziùnne, mànche se fasciavànt l'adunàte, rénnh (CMIII3) - CO 'on (ne) faisait même pas d'exercices militaires, on (ne) faisait même pas même pas de rassemblement, rien'

49. su ò va pa, su ò va pas, se scàrepe accussì me piasciùnte pa, lu cauzùnne accullì manche lo uótte (CFIII4) - CO 'ça (ne) va pas, ça (ne) va pas, les chaussures comme ça (ne) me plaisent pas, le pantalon comme ça, il en veut même pas'

L'analyse des tournures négatives avec un mot-N nominal permet de dégager des tendances très nettes : 1) l'alternance systématique de mangùnne (nessuno, (ne) personne) en faetano et de la variante gnun/gnùnnh en cellese ; 2) l'absence de pa lorsque le mot-N est en position préverbale (ex. 50-53) ; 3) la présence de $p a$ lorsque le mot-N est placé après le verbe (ex. 54-57) (Martino, 2011 : 38); en outre, le marqueur négatif apparaît plus fréquemment en combinaison avec le mot-N renne :

50. Decchìrre se mengiàve mangùnne i parlàve [...] (G\&P : 54) - CE (variété faetana)

Decchìre se mengiàve gnùnnh i parlàve [...] (G\&P : 55) - CE (variété cellese)

'Pendant le repas, personne (ne) parlait'

51. mancúnne u munne gli-é megliàue de ti (FFIII4) - CT 
gnùn a lu munne gli-étte megliàue de ti (CFIII7) - CT

'personne au monde n'est meilleur que toi'

52. mancùnne i quanàje a mancùnne (FMII1) - CT

gnùn i quanàje a gnùn (CFIII4) - CT

'personne ne connaît personne'

53. illé gnùn i savìve natà (CFII2) - CO

'là, personne (ne) savait nager'

54. m'éve perdì ma a-gn-ave pa mangùnne (FFII1) - CT

'je m'étais perdu et (il n') y avait personne'

55. Denghjé lu 1943, decchìrre denghjé u munne s'accappive pa rénne [...] (G\&P : 100) - CE

'En 1943, quand partout dans le monde on ne comprenait rien'

56. G'é pa di rénne a mancùnne (FFIII4) - CT

$G$ 'é pa ditte $[\mathrm{ditt}] /($ di) rénne a gnùn $(\mathrm{CFI} 3)-\mathrm{CT}$

'j(e n') ai rien dit à personne'

57. a Faìte a premavére i giuccùnte u miéce a la vì ó fa pa rénne (FFII5) - CO

'à Faeto, au printemps, ils jouent dans la rue, ça (ne) fait rien'

Toutefois, lorsque le mot-N est postposé au verbe, la présence du marqueur demeure facultative (Martino, 2011 : 11). Les cas d'omission du marqueur dans ce genre d'énoncé constituent une tendance secondaire :

58. g'è di rénne a mancùnne (FMI1) - CT

'j(e n') ai rien dit à personne'

59. m'éve perde [perdə]/(perdì) é a-gn-ave mangùnne (FFI1) - CT

me séve sperdì é ave gnùn (CFIII4) - CT

'je m'étais perdu et (il n') y avait personne'

60. gljó ma te lo disce faitàre, [sen'no]/(sennù) i sèrve a rénne (FFI2) - CO

'oui, mais tu le dis en faetano, sinon ça (ne) sert à rien'

61. ajére i-àtte féje rénnh (CFII1) - CO

'hier, il (n') a rien fait'

62. a-gn-àve quase mancùnn (FFI2)- $\mathrm{CO}$

'(il n') y avait presque personne'

Une observation doit être faite à propos de l'alternance mancùnne/gnùn. Les locuteurs cellesi ont parfois choisi mancùnne au lieu de gnùn. Certains de ces locuteurs se déclarent peu compétents en FP.

$P a$ est omis dans les quelques énoncés où un mot-N nominal et un mot-N adverbial co-occurrent :

63. g'é màje di rénne a mancùnne (FMI2) - CT

'j(e n') ai rien dit à personne'

64. é màje succedì rénne (CMII2) - $\mathrm{CO}$

'il (ne) s'est jamais rien passé'

65. se desidere ré méje pecché é tutte ['pronto]/(prónte/prónde ) (CFIII4) - CO

'on (n') a plus envie de rien parce qu'on a tout sous la main'

66. ghniùnnh lò purtavànt a mengìje, lò purtavànt mànche rénnh (CMIII3) - $\mathrm{CO}$

'personne (ne) leur portait à manger, ils (ne) leur portaient rien du tout'

Cette description du fonctionnement de la négation en faetano-cellese ne fait pas ressortir le fait que les taux de chaque type de structure engendrent différentes configurations de données selon le type de corpus envisagé. En guise de synthèse, nous proposons une analyse croisée entre les fréquences d'apparition du marqueur tout seul, des seuls mots-N ou de leur combinaison selon les différentes typologies de corpus. 


\subsection{Synthèse comparative des résultats de l'analyse rapportée aux différentes typologies de données}

Les textes écrits permettent de dresser un tableau assez homogène. La plupart des énoncés négatifs sont des exemples de négation totale avec pa. Les cas de négation ne s'appuyant que sur les adverbiaux chjù et manghe sont peu représentés, tandis que l'occurrence des formes combinées pa màje et pa manghe est légèrement plus importante. Aucune différence ne se dégage entre la variété faetana et la variété cellese, sauf l'alternance systématique des mots-N nominaux mancùnne et gnùnnh. Ce n'est peut-être pas un hasard si le corpus écrit ne reflète pas une donnée pourtant évidente dans le corpus de traduction, à savoir le fait qu'à l'emploi de chjù en faetano correspond l'usage quasi systématique de pa ou pa méje en cellese. En effet, la visée homogénéisante du processus de standardisation du FP à travers l'écriture efface la disparité des rapports de force entre les deux parlers francoprovençaux. Dans les représentations linguistiques des locuteurs, le cellese est réputé plus conservateur que le faetano qui aurait, au contraire, un facies plus italianisé, ou "napolétanisé » comme le disent les locuteurs eux-mêmes, à cause des échanges plus denses et de longue date que Faeto entretient avec les communautés italo-romanes environnantes. Mais malgré sa plus grande perméabilité au diasystème IT-dialectes italo-romans, le faetano est aussi la variété parlée par la communauté la plus nombreuse, celle qui dispose de plus de services commerciaux et qui est dotée d'écoles. Le faetano fait alors office de variété " dominante » par rapport au cellese. D'un point de vue idéologique, on cherche à perpétuer l'idée qu'il existe des différences de prononciation et de vocabulaire entre faetano et cellese, ce qui est aussi le cas pour quelques formes lexicales. Mais l'usage généralisé de chjù dans les textes écrits est un indice, sans doute faible mais peut-être révélateur, susceptible d'annoncer un processus de progressive dissolution des différences entre les deux variétés, au sein duquel la variété faetana joue le rôle d'élément moteur.

Les données du corpus écrit traduisent le plus directement l'influence de facteurs extrasystémiques. En revanche, le corpus de traduction met en cause des facteurs intersystémiques, tels que les phénomènes d'emprunt, d'interférence et de convergence entre les langues en contact. La traduction de l'IT au FP a sans doute provoqué des distorsions sur les productions obtenues en FP, mais il nous semble que l'un des effets de telles distorsions a été celui d'avoir amplifié une tendance bien plus faible dans les textes écrits et dans le discours oral semi-spontané, à savoir l'emploi d'un mot-N comme unique élément de négation. Le fait que la structure $\mathrm{V}+$ mot- $\mathrm{N}$ caractérise avant tout les données provenant de la traduction de phrases italiennes amène à penser que le contact avec l'IT doit y jouer un rôle qui, pourtant, n'est pas facile à identifier. On pourrait supposer que l'effacement du pa résulte d'un processus de rapprochement du FP vers l'IT, peut-être favorisé du fait que le mot-N se trouve en IT aussi en position postverbale, ce qui permet de maintenir un trait syntaxique stable du système de la négation en FP, tout en supprimant un élément ressenti comme typiquement francoprovençal. Mais si le faetano-cellese se distingue du FS par la co-occurrence du marqueur pa et du mot-N d'une part, d'autre part il fonctionne comme le français parlé non standard qui prévoit une négation phrastique avec la série des forclusifs pas, point, plus, jamais, personne, rien. En outre, d'autres variétés diatopiques de français permettent, sous différentes modalités, la coprésence du marqueur et d'un mot- $\mathrm{N}$, tels que le créole martiniquais, le français québécois, le picard, ce qui complique davantage l'analyse ${ }^{20}$. Rappelons que les patois de la France, de la Suisse romande et de la Vallée d'Aoste offrent aussi de rares cas de co-occurrence du marqueur négatif et d'un mot-N (\$3.1). Le fait que le polymorphisme observé en faetano-cellese soit attesté dans d'autres variétés de français amène à l'expliquer comme résultant de dynamiques intrasystémiques.

En ce qui concerne les données de parole semi-spontanée, elles se conforment en partie aux données écrites par leur homogénéité : la plupart des énoncés ont la structure Vpa (76\% des énoncés recueillis). Le recours à la structure $p a+$ mot-N constitue une tendance secondaire, tandis que les cas de négation ne s'appuyant que sur le seul mot-N sont sporadiques. Les productions semi-spontanées font aussi apparaître des faits qu'on peut ramener à l'influence de l'IT, tels que l'emploi de la négation renforcée pa proprje ou la possibilité d'antéposer manghe au verbe.

Tous types de corpus confondus, il se dégage des tendances qui, en se croisant, semblent avoir pour effet d'augmenter la productivité de la variation elle-même. L'emploi du marqueur en présence d'un mot-N 
est facultative, et cela dans des contextes identiques. La présence du marqueur est quasi systématique avec les mots-N màye, manghe, méje et renne, mais presque exclue avec chjù. À propos des combinaisons adjacentes pa màje, pa méje, pa manghe, il est difficile de dire si l'expression du marqueur est favorisée par la présence d'un verbe conjugué à la forme composée ou plutôt par le type de mot-N. Les structures négatives avec un mot-N tout seul apparaissent surtout dans la traduction en FP de phrases données en IT. La forme de certains mots-N et les deux cas où manghe est placé avant le verbe sont une manifestation évidente du contact linguistique. L'effacement du marqueur pourrait s'interpréter comme une sorte d'alignement du FP sur l'IT, celui du mot-N comme une surextension de la structure Vpa.

Quant à la corrélation avec les classes d'âge, qui implique de manière indirecte le paramètre de la compétence linguistique $(\S 4.1)^{21}$, le résultat le plus significatif concerne la I ${ }^{\text {ème }}$ classe. En effet, par rapport au corpus de traduction seulement, les jeunes et jeunes adultes se caractérisent par l'emploi d'un mot-N tout seul $\left(63 \%\right.$ contre $35 \%$ pour la II ${ }^{\text {ème }}$ classe et $45 \%$ pour la III $\left.{ }^{\text {ème }}\right)$. D'après ce résultat, l'éventuel

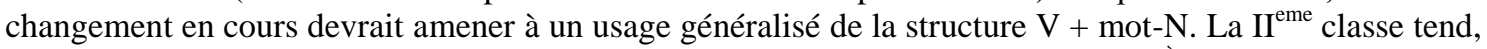
en revanche, à employer le plus fréquemment la structure $p a+$ mot-N. Dans la III ${ }^{\text {ème }}$ classe, les taux des deux types de structures sont presque identiques. Le nombre exigu d'énoncés d'oral semi-spontané avec un mot-N ne permet d'établir aucune corrélation avec les classes d'âge et, de plus, l'écart entre les énoncés avec et sans pa est toujours très réduit.

En définitive, pour ce qui est du polymorphisme syntaxique analysé, si d'un côté il ne semble pas résulter du contact linguistique, de l'autre côté, les énoncés de traduction font supposer que le contact avec l'IT pourrait quand même y jouer un rôle. Force est alors de laisser en suspens, avant de pousser plus loin l'analyse, la compréhension de la nature et des causes de la variabilité intrinsèque, déjà intéressante en soi, se produisant au sein d'une langue obsolescente telle que celle qui a fait l'objet de cette étude.

\section{Remarques conclusives}

L'étude de cas présentée touche une zone de variabilité potentielle du système gallo-roman et en même temps un point de résistance du système faetano-cellese aux pressions assimilatrices des autres langues de contact. Le faetano-cellese constitue un système linguistique résultant de l'intrication de facteurs intrasystémiques et intersystémiques, qui acquièrent, tour à tour, plus ou moins d'importance selon le phénomène linguistique considéré. C'est pourquoi, il devient un système intéressant pour tester les modèles et les modalités de changement dans une langue en danger et dans un contexte de langues en contact. Partant du principe que, dans une situation de contact intense et de longue durée, la syntaxe même peut être empruntée, notre tentative de rechercher les symptômes d'un éventuel changement induit par le contact dans une «frontière » poreuse, comme aurait pu l'être le contexte de co-occurrence d'un marqueur négatif et d'autres mots-N, s'est heurtée à l'enchevêtrement de facteurs intralinguistiques et interlinguistiques. En effet, lorsqu'on tourne son regard vers la forme des mots-N, et encore plus si l'on élargit la perspective à l'ensemble du lexique faetano-cellese et à plusieurs aspects de la syntaxe de la phrase toute entière, la convergence vers le diasystème IT-dialectes italo-romans se révèle dans toute son évidence. En revanche, lorsque le regard s'arrête sur la syntaxe de la phrase négative, tout particulièrement sur la position postverbale du marqueur et des mots- $\mathrm{N}$, on doit se replier sur le système gallo-roman. Bien qu'une certaine variabilité dans l'expression du marqueur négatif en présence d'un mot-N ait été remarquée, la difficulté majeure demeure celle de l'expliquer. D'un côté, les phénomènes de variation considérés se retrouvent dans plusieurs variétés de français, de l'autre, on sait que toute variation observable en synchronie ne doit pas forcément déclencher un changement et qu'il existe des «variations stables » pouvant perdurer tout au long des siècles (la négation en FS et en français parlé non standard en est un exemple) (Blanche-Benveniste, 2007 : 19). S'agissant d'une variabilité qui se produit dans une langue en danger, il va presque de soi qu'on en recherche les causes dans le contact avec les langues dominantes. Toutefois, les résultats de l'analyse nous forcent à laisser ouverte la voie à des explications plus complexes, qui mettent en jeu la recherche de motivations internes de la variabilité, et éventuellement du changement, ayant lieu au sein d'un système linguistique obsolescent. 
Pour conclure, le faetano-cellese offre l'exemple d'une langue où s'entremêlent des strates synchroniques stables et instables (Houdebine, 1985), les unes plus ou moins résistantes au contact et les autres fortement perméables aux autres langues, ce qui invite à réfléchir davantage sur la lenteur des processus d'obsolescence et sur des langues qui, tout en mourant, laissent «vivre » certains de leurs traits les plus marquants.

\section{Références bibliographiques}

Aikhenvald, A.Y. (2012). Language contact in language obsolescence. In C. Chamoreau et I. Léglise (éds.), Dynamics of contact-induced language change. Berlin : Mouton de Gruyter, 77-109.

Anello, T. (2013/2104). Varietà (s)confinate e confinanti: interconnessioni umane, ambientali e sociali nel francoprovenzale di Faeto e Celle di San Vito. Thèse de Master, Université de Naples Federico II.

Ascoli, G. I. (1878). Schizzi Franco-Provenzali. Archivio Glottologico Italiano, 3, 61-120.

Bavoux, C. (2003). Quand des langues de grande proximité sont en contact : modalités d'existence et de coexistence. In J. Billiez (éd), Contacts de langues. Modèles, typologies, interventions. Paris : L'Harmattan, 25-35.

Bisang, W. (2004). Dialectology and typology - An integrated perspective. In B. Kortmann (éd.), Dialectology meets typology. Dialect grammar from a cross-linguistic perspective. Berlin : Walter de Gruyter, 11-45.

Blanche-Benveniste, C. (2007). Le français parlé au $21^{\text {ème }}$ siècle. Réflexions sur les méthodes de description: système et variations. In M. Abecassin, L. Ayosso et É. Vialleton (éds.), Le français parlé au XXI siècle : normes et variations géographiques et sociales, vol. I. Paris : L'Harmattan, 17-39.

Chambon, J.-P. et Greub, Y. (2000). Données nouvelles pour la linguistique galloromane : les légendes monétaires mérovingiennes. Bulletin de la Société de linguistique de Paris, 95, 147-182.

Chamoreau, C. et Léglise, I. (éds.). (2012). Dynamics of contact-induced language change. Berlin : Mouton de Gruyter.

Corblin, F. et Tovena, L. M. (2003). L'expression de la négation dans les langues romanes. In D. Godard (éd.), Les langues romanes : problèmes de la phrase simple. Paris : CNRS Éditions, 281-343.

Dagnac, A. (2015). 'Pas', 'mie', 'point' et autres riens : de la négation verbale en picard. In Pitar M. et Goes J. (éds.), La négation. Études linguistiques, pragmatiques et didactiques. Arras : Artois Presses Université, 129-152.

Dahl, Ö. (1979). Typology of sentence negation. Linguistics, 17, 79-106.

Dahl, Ö. (2010). Typology of negation. In L. R. Horn (éd.), The expression of negation. Berlin : Mouton de Gruyter, 9-38

Déprez, V. (2003). Concordance négative, syntaxe des mots-N et variation dialectale. Cahier de Linguistique Française, 25, 97-118.

Diémoz, F. et Kristol, A. (2012). L'Atlas linguistique audiovisuel du francoprovençal valaisan et les défis du polymorphisme. In D. Kattenbusch et F. Tosques (éds.), 20 Jahre digitale Sprachgeographie. 20 ans de géolinguistique numérique. 20 anni di geolinguistica digitale. 20 años de geografía lingüística digital. Actes du colloque de Berlin (Humboldt-Universität, 2-3 novembre 2012). Berlin : Humboldt-Universität, Institut für Romanistik, 163-183.

Dorian, N. (1977). The problem of the semi-speaker in language death. The International Journal of the Sociology of Language, 12, 23-32.

Dorian, N. (1981). Language death. The life cycle of a Scottish Gaelic dialect. Philadelphia : University of Pennsylvania Press.

Dryer, M. S. (2011a). Order of negative morpheme and verb. In M. S. Dryer et M. Haspelmath (éds.), The World Atlas of Language Structures Online (chapter 143). Munich : Max Planck Digital Library. http://wals.info/chapter/143 
Dryer, M. S. (2011b). Position of negative morpheme with respect to subject, object and verb. In M. S. Dryer et M. Haspelmath (éds.), The World Atlas of Language Structures Online (chapter 144). Munich : Max Planck Digital Library. http://wals.info/chapter/144

Dryer, M. S. (2011c). Negative morphemes. In M. S. Dryer et M. Haspelmath (éds.), The World Atlas of Language Structures Online (chapter 112). Munich : Max Planck Digital Library. http://wals.info/chapter/112

Epps, P. (2012). On form and function in language contact: A case study from the Amazonian Vaupés region. In C. Chamoreau et I. Léglise (éds.), Dynamics of Contact-Induced Language Change. Berlin : Mouton de Gruyter, 195-230.

Genovese, A. et Pastore, N. (2011). Cunte d'ajére é d'avi 4. Il Provenzale.

Haspelmath, M. et al. (éds.). (2005). The World Atlas of Language Structures. Oxford : Oxford University Press. http://wals.info/

Hasselrot, B. (1938/1939). Sur l'origine des adjectifs possessifs nostron et vostron en francoprovençal. Studia neophilologica, 11, 62-84.

Hasselrot, B. (1974). Adieu au francoprovençal. Revue de linguistique romane, 38, 265-275.

Heine, B. et Kuteva, T. (2003). On contact-induced grammaticalization. Studies in Language, 27, 529-572.

Houdebine, A.-M. (1985). Pour une linguistique synchronique dynamique. La Linguistique, 21, 7-36.

Jespersen, O. (1917). Negation in English and other languages. København : Høst.

Léglise, I. et Chamoreau, C. (2013). Variation and change in contact settings. In I. Léglise et C. Chamoreau (éds.), The interplay of variation and change in contact settings. Amsterdam : John Benjamins Publishing Company, 120.

Martino, A. (2011). Reliquie francoprovenzali nella parlata di Celle di San Vito (Foggia). LINCOM Studies in Romance Linguistics, 67, Munich : LINCOM Europa.

Matras, Y. (2012). An activity-oriented approach to contact-induced language change. In C. Chamoreau et I. Léglise (éds.), Dynamics of Contact-Induced Language Change. Berlin : Mouton de Gruyter, 17-32.

Matthey, M.et Meune M. (2012). Anthologie de textes romands en francoprovençal. In M. Matthey et M. Meune (éds), Le francoprovençal en Suisse. Genèse, déclin, revitalisation, Revue transatlantique d'études suisses, 2, 107-123.

Meillet, A. (1912). L'évolution des formes grammaticale. Scientia, 12, 384-400. [Aussi dans A. Meillet (1926). Linguistique historique et linguistique générale. Paris : H. Champion, p. 130-148.]

Melillo M. (1959). Intorno alle probabili sedi originarie delle colonie franco-provenzali di Celle e Faeto. Revue de linguistique romane, $23,1-34$.

Meyerhoff, M. et N. Nagy. (2008). Social lives in language - Sociolinguistics and multilingual speech communities, Amsterdam : Benjamins.

Miestamo, M. (2011). A typological perspective on negation in Finnish dialects. Nordic Journal of Linguistics, 34, 83-104.

Minichelli, Vincenzo. 2002 [1994]. Dizionario francoprovenzale di Celle di San Vito e Faeto, 2ème édition, Présentation de Tullio Telmon, Alessandria : Edizioni dell'Orso.

Moseley, C. (éd). 2010. Atlas des langues en danger dans le monde, $3^{\mathrm{e}}$ édition. Paris : Éditions UNESCO. www.unesco.org/culture/en/endangeredlanguages/atlas

Nagy, N. (2011). Lexical change and language contact: Faetar in Italy and Canada. Journal of Sociolinguistics, 15, 366-382.

Puolato, D. (2010). Lingua, dialetto e identità: percezioni e rappresentazioni della enclave francoprovenzale di Faeto. Bollettino Linguistico Campano, 18, 43-79.

Puolato, D. (2011). Intrecci storici, linguistici ed identitari nella minoranza francoprovenzale di Celle di San Vito. Bollettino Linguistico Campano, 19/20, 91-149. 
Puolato, D. (2013). Les appellations de la langue minoritaire à Faeto et à Celle di San Vito (Pouilles) : valeurs identitaires et idéologiques. In C. Alén Garabato (éd). Gestion des minorites linguistiques dans l'Europe du XXI siècle, Limoges : Lambert-Lucas, 179-191.

Puolato, D. (2016). L'(in)sécurité linguistique et les locuteurs francoprovençaux de l'Italie du Sud. In G. Agresti et H. Giordan (éds), Les droits linguistiques entre représentations, idéologies et politiques linguistiques. Quels rapports, quelle(s) intervention(s)?, Coll. Lingue d'Europa e del Mediterraneo, Rome: Aracne editrice (sous presse).

Sportello Comunale della Lingua Francoprovenzale (SLF). (2007a). Dizionario Francoprovenzale-Italiano / ItalianoFrancoprovenzale di Faeto. Foggia : L'Editrice.

Sportello Comunale della Lingua Francoprovenzale (SLF). (2007b). Grammatica Francoprovenzale di Faeto. Foggia, L'Editrice.

Sportello Comunale della Lingua Francoprovenzale (SLF). 2005. Glossario con rimario in francoprovenzale di Faeto, Foggia: L'Editrice.

Stich, D. (1998). Parlons francoprovençal. Une langue méconnue. Paris : L’Harmattan.

Tuaillon, G. (1983). Le francoprovençal : progrès d'une définition. Saint Nicolas : Centre d'études francoprovençales René Willien (aussi in Travaux de Linguistique et de Littérature, 10, 1972, 293-339).

Tuaillon, G. (1988). Le francoprovençal, langue oubliée. In G. Vermes (éd.), Vingt-cinq communautés linguistiques de la France, 1, Langues régionales et langues non territorialisées. Paris : L’Harmattan, 188-207.

van der Auwera, J. (2009). The Jespersen cycles. In E. van Gelderen (éds.), Cyclical change. Amsterdam : Benjamins, 35-71.

van der Auwera, J., Vossen, F. et Devos, M. (2013). Le cycle de Jespersen à trois ou quatre négation. In J. François, P. Larrivée, D. Legallois et F. Neveu (éds.), La linguistique de la contradiction. Bern : Peter Lang, 19-30.

\footnotetext{
${ }^{1}$ L'interrelation entre variation, changement et contact linguistiques est au centre des travaux de Meyerhoff et Nagy (2008), Chamoreau et Léglise (2012), Léglise et Chamoreau (2013), entre autres.

${ }^{2}$ Pour expliquer le changement résultant du contact, Matras (2012) propose une analyse croisée entre contexte social, dynamiques conversationnelles et intentions communicatives, et fonction des structures ou catégories données.
}

3 Voir l'Atlas des langues en danger dans le monde (Moseley, 2010), disponible à la page web http://www.unesco.org/culture/languages-atlas/fr/atlasmap.html.

${ }^{4}$ Il existe des exceptions à cette construction. Par exemple, en Afrikaans, la phrase négative se construit avec deux marqueurs négatifs identiques en position postverbale (Dahl, 2010:20); en portugais brésilien une même particule négative se place à la fois avant et après le verbe (van der Auwera, 2009 : 80).

${ }^{5}$ Quoique rares, il existe aussi des cas de négation verbale à trois négateurs (notamment dans le bantou et le malayopolynésien) ou à quatre négateurs. En outre, l'absence d'un marqueur explicite de négation, elle aussi rare, reste à l'écart du cycle de Jespersen (van der Auwera, Vossen et Devos, 2013).

${ }^{6}$ La catégorie des mots- $\mathrm{N}$ renvoie à un ensemble de mots hétérogènes quant à leurs propriétés syntaxiques et sémantiques, ce qui a donné lieu à de multiples approches d'analyse. Le terme « mots-N » peut en effet désigner des expressions proprement négatives autant que des items à polarité négative, qui doivent être légitimés par un déclencheur approprié. Selon la terminologie de Corblin et Tovena (2003), les mots-N désignent à la fois les expressions qui englobent morphologiquement le morphème négatif (nessuno en IT) et des expressions associées aux arguments d'un prédicat et qui s'interprètent comme des quantifieurs négatifs (personne en français). On distingue entre mots-N adverbiaux (comme jamais ou plus) et nominaux (c'est le cas de personne, rien, etc.).

${ }^{7}$ Selon la théorie traditionnelle, la séparation entre le FP et le domaine d'oïl a commencé vers le milieu du $8^{\mathrm{e}}$ siècle. Cependant, de nouvelles données issues des légendes des monnaies mérovingiennes permettent de dater déjà dès les $6^{\mathrm{e}} / 7^{\mathrm{e}}$ siècles une série d'évolutions phonétiques propres au domaine francoprovençal.

${ }^{8}$ Le critère le plus fiable pour circonscrire le domaine francoprovençal est l'évolution des voyelles finales atones : est $\mathrm{FP}$ « tout parler où $-\mathrm{AS},-\mathrm{AT}>e,-\mathrm{a}>a$, palatale $+\mathrm{a}>i$, et où -o est conservé », en tenant compte, d'ailleurs, que « le 
critère fourni par - $i$ final» (VACCA > la vatsi, la vashi) a «une importance toute spéciale », «puisque le francoprovençal en a l'exclusivité » (Hasselrot, $1974: 266$ ).

${ }^{9}$ Plus précisément, «la palatalisation de $k$ et $g+a$, génératrice à son tour du développement de $a$ en $\varepsilon$ (loi de Bartsch)» (Hasselrot, $1974: 270)$.

${ }^{10}$ Sources consultées : 1) «Petit florilège de la littérature francoprovençale » (Stich, 1998);2) « Anthologie de textes romands en francoprovençal» (Matthey et Meune, 2012) ; 3) http://patoisvda.org, http://www.patoisdenendaz.ch, http://www.patoisvaudois.ch, http://www.patoisneuchatelois.net, http://patwe.ch, http://nontra.lingua.free.fr, http://www.langue-savoyarde.com.

${ }^{11}$ Énoncés repérés : Ma fei pa rente (Mais peu importe), Donnas ; «Ivrède po la porta a gneun » (« N'ouvrez la porte à personne »), Arvier ; "Regalade-vó é lèichade pa pédre ren!»(Régalez-vous bien et ne laissez rien), Aoste ; El n'en reste pas més ren... (Il n'en reste plus guère), Lyon; no n'in pâ méi po fran ontin (il n'y en plus pour très longtemps), Nedaz (F); Y ne porra pa guéro la garda (Il ne pourra guère la garder), Genève.

12 Il s'agit d'un concours de récits en FP sur des sujets qui évoquent la vie, la culture et les traditions des communautés francoprovençales apuliennes. Il est organisé par «Il Provenzale », le périodique des minorités francoprovençales de Faeto et Celle.

${ }^{13}$ L'adverbe mai constitue une exception : il peut aussi accompagner un autre mot-N en position préverbale (nessuno mai / mai nessuno dovrà saperlo), mais lorsqu'il existe d'autres mots-N qui suivent le verbe, il ne peut pas occuper tout seul la position préverbale. Pour d'autres approfondissements, voir Corblin et Tovena (2003).

${ }^{14}$ Pour une analyse sociolinguistique très fine du corpus de traduction, voir Anello (2014).

${ }^{15}$ La transcription orthographique des corpus de traduction et d'oral semi-spontané a été effectuée par deux locuteurs-informateurs, l'un faetano et l'autre cellese, dont nous maintenons la graphie et les variantes éventuelles. Les textes analysés sont très riches en code-switching, code-mixing, emprunts et interférences, mais il est toutefois fort délicat de trancher parmi ces phénomènes, tellement ils sont constitutifs du parler faetano-cellese. Dans le présent article, nous ne nous limitons qu'à signaler, par le moyen de transcriptions phonétiques, les phénomènes de code-switching notamment, tout mot non attesté dans les dictionnaires consultés (Minichelli, 2002 ; Martino, 2011 ; SLF, 2005 et 2007a) et les mots attestés mais dont la réalisation dénote une prononciation dialectale italianisée. Ces mots sont mis entre parenthèses après la transcription phonétique.

${ }^{16}$ Dans le recueil de contes analysé, la différence entre le faetano et le cellese ne se manifeste qu'au niveau lexical ou phonético-graphique. En l'absence de différences concernant la syntaxe de la phrase négative dans la représentation écrite des deux variétés, nous ne reproduisons que la version faetana des énoncés tirés du recueil (siglé G\&P).

${ }^{17}$ Ce sigle indique le lieu d'enquête (Faeto ou Celle), le sexe du locuteur (Femmina ou Maschio), la classe d'âge (I, II, III) et le numéro de l'interview.

${ }^{18} \mathrm{La}$ forme autochtone est pettócche, mais la variante dialectale pecché [pək'ke] est très diffusée.

${ }^{19}$ Forme atone du pronom personnel sujet de la $1^{\text {ère }}$ personne du pluriel (Martino, $2011: 32$; SLF, 2007b : 156).

${ }^{20}$ Le créole martiniquais requiert obligatoirement la présence du marqueur $p a$ dans une phrase déclarative négative qui contient un ou plusieurs mots-N (Déprez, 2003). En français québécois le marqueur peut co-occurrer avec un mot-N, mais, comme en IT, cette co-occurrence n'est possible que lorsque le mot-N est posposé au verbe (Déprez, 2003). Le picard admet aussi la co-occurrence du marqueur négatif avec des mots-N, mais le marqueur peut être aussi présent au cas où le mot-N se trouve en position de sujet préverbal (Dagnac, 2015).

${ }^{21}$ Dans cette étude les compétences en FP n'ont été «mesurées » que de façon approximative, tenant compte d'indices tels que le nombre des phrases traduites en FP et la compétence lexicale du FP en production et en réception. Une analyse plus approfondie des compétences langagières des locuteurs, tant en IT qu'en FP, et de leurs attitudes linguistiques pourrait peut-être aboutir à des résultats différents. 